\title{
Subserosal recurrence without mucosal involve- ment diagnosed 5 years after endoscopic sub- mucosal dissection for early rectal cancer
}
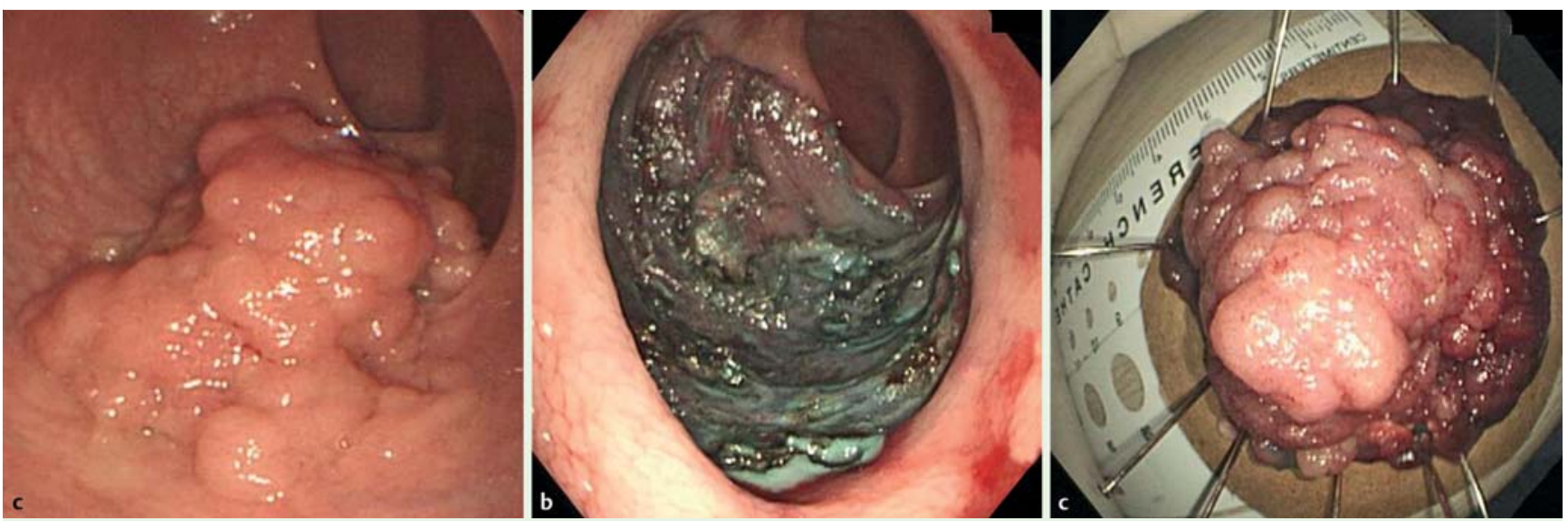

Fig. 1 Colonoscopic findings. a A 4-cm, nodular, mixed-type, laterally spreading tumor in the rectum. b Endoscopic submucosal dissection just above the muscular layer toward the proximal side of the tumor. c Macroscopic appearance of the resected specimen.

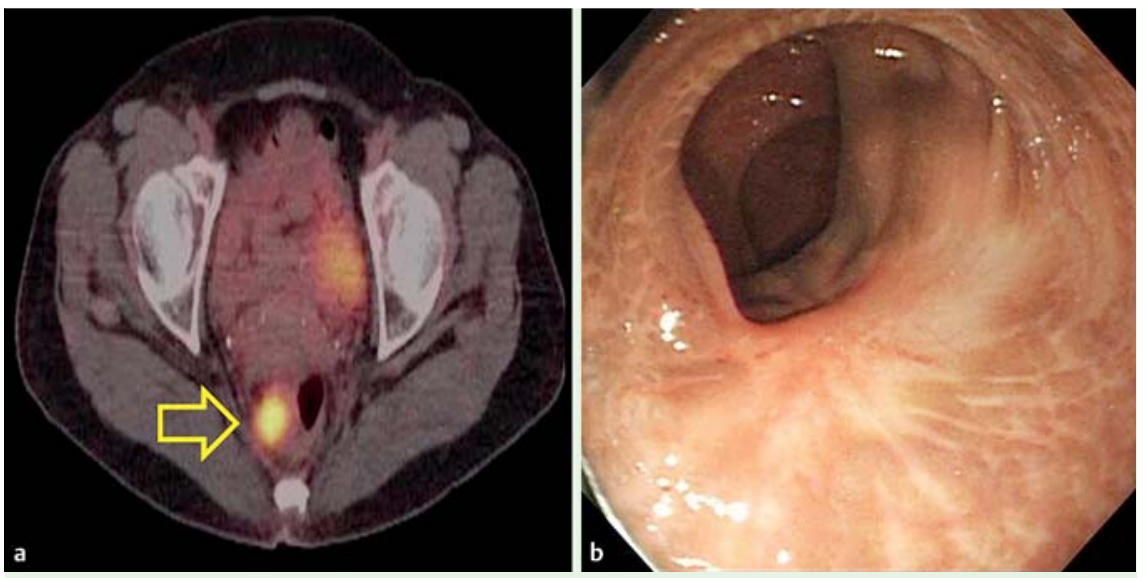

Fig. 2 Surveillance studies 5 years after endoscopic submucosal dissection. a Positron emission tomography-computed tomography revealed a hypermetabolic mass in the right wall of the rectum, with perirectal invasion. $\mathbf{b}$ Changes in the scar were found at the previous polypectomy site on colonoscopy.

A 61-year-old woman underwent successful en bloc endoscopic submucosal dissection (ESD) for the management of a 4-cm, nodular, mixed-type, laterally spreading tumor located $11 \mathrm{~cm}$ from the anal verge ( $\bullet$ Fig. 1). Although the depth of submucosal invasion was $3000 \mu \mathrm{m}$, the patient refused additional surgical treatment. Three weeks after ESD, the patient developed an extraperitoneal pelvic abscess, which was caused by delayed rectal perforation and required percutaneous drainage.

During 5 years of post-ESD follow-up, surveillance colonoscopy showed no recurrence at the ESD scar ( $\bullet$ Fig. 2). However, surveillance positron emission tomography computed tomography conducted 5 years after the initial endoscopic treatment revealed a newly formed, intensely hypermetabolic mass in the right wall of the rectum, with perirectal invasion ( Fig. 2a). Laparoscopic low anterior resection with lymph node dissection was performed. Examination of the specimen revealed a $4.5 \times 3.5 \mathrm{~cm}$ subserosal mass, located $2 \mathrm{~cm}$ distal to the previous endoscopic resection scar, with no evidence of mucosal changes ( Fig.3). Pathological examination of the specimen showed a moderately differentiated T3NO adenocarcinoma with lymphatic and perineural invasion. The recurrent rectal cancer had invaded the subserosal layer, but the mucosal layer was intact and not involved (ه Fig.4).

The recurrence pattern after endoscopic resection of colorectal tumors is usually restricted to intramural tissues, with a mucosal lesion at the previous endoscopic mucosal resection or ESD site [1-3]. The exact mechanism of local recurrence in this patient is unknown, although we suspect that recurrence was caused by implantation of residual tumor cells in the subserosal area after ESD as a result of delayed rectal perforation and abscess formation. We suggest that this case represents an unusual pattern of local recurrence after ESD, as opposed to a new lesion. Thorough long-term followup with multimodal evaluations is therefore necessary after ESD, in order to detect any local recurrence.

Endoscopy_UCTN_Code_CPL_1AJ_2AD

Competing interests: None

\section{Sung Uk Bae, Woon Kyung Jeong, Ok Suk Bae, Seong Kyu Baek}

Department of Surgery, School of Medicine, Keimyung University and Dongsan Medical Center, Daegu, Korea 


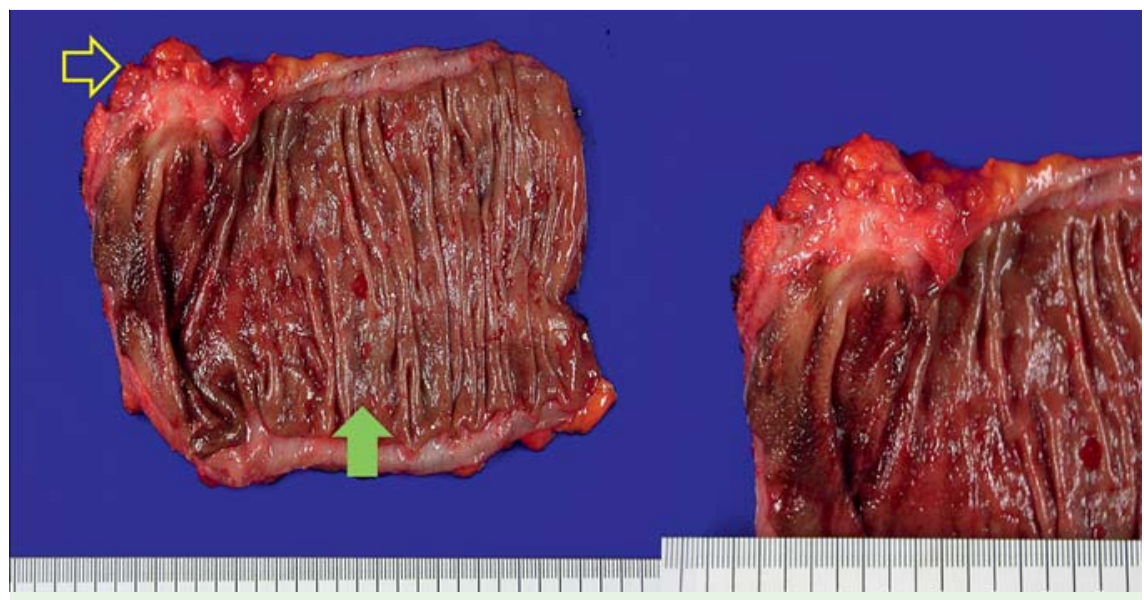

Fig. 3 Examination of the specimen showed a $4.5 \times 3.5 \mathrm{~cm}$ subserosal mass (empty arrow) located $2 \mathrm{~cm}$ distal to the previous endoscopic resection scar (filled arrow), with no evidence of mucosal changes.
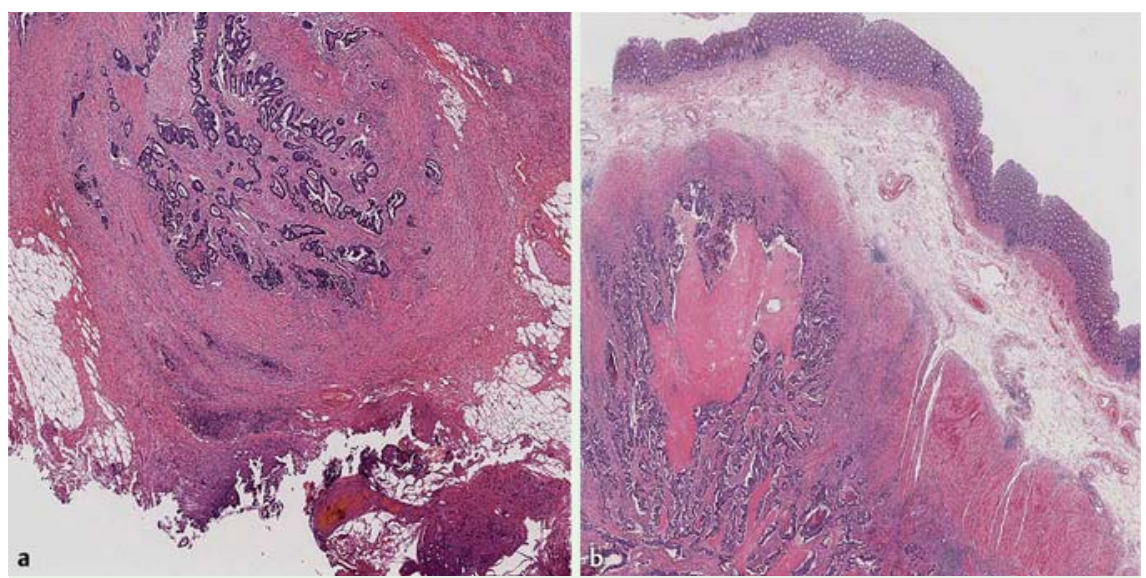

Fig.4 a Microscopic examination revealed a moderately differentiated adenocarcinoma with lymphatic and perineural invasion. $\mathbf{b}$ The tumor had invaded the subserosal layer, but the mucosal layer was intact.

\section{References}

1 Oka S, Tanaka S, Kanao $H$ et al. Current status in the occurrence of postoperative bleeding, perforation and residual/local recurrence during colonoscopic treatment in Japan. Dig Endosc 2010; 22: 376 - 380

2 Seo GJ, Sohn DK, Han KS et al. Recurrence after endoscopic piecemeal mucosal resection for large sessile colorectal polyps. World J Gastroenterol 2010; 16: 2806 - 2811

3 Tanaka S, Haruma K, Oka S et al. Clinicopathologic features and endoscopic treatment of superficially spreading colorectal neoplasms larger than $20 \mathrm{~mm}$. Gastrointest Endosc 2001; 54: 62-66

\section{Bibliography}

DOI http://dx.doi.org/

10.1055/s-0034-1391495

Endoscopy 2015; 47: E166-E167

(c) Georg Thieme Verlag KG

Stuttgart · New York

ISSN 0013-726X

Corresponding author

Seong Kyu Baek, MD

Department of Surgery

School of Medicine

Dongsan Medical Center

Keimyung University

194 Dongsan-Dong, Jung-Gu

700-712 Daegu

Republic of Korea

Phone: +82-53-2507322

sgbeak@dsmc.or.kr 